\title{
A note on a result of K.D. Magill, Jr
}

\section{Eric Warren}

The result of Magill concerning differentiable maps on the reals; namely, that all automorphisms of this semigroup are inner; is shown to be untrue in the case of analytic maps on the complexes. Also a characterization of the inner automorphisms of the analytic maps is given.

Let $R$ denote the reals and let $D(R)$ denote the set of all (finitely) differentiable maps from $R$ to $R$. In [2] Magill proves that every automorphism of $D(R)$ is inner ([2, Theorem 2.1]). He also shows that the set of inner automorphisms of $D(R)$ is isomorphic to the set of all strictly monotonic homeomorphisms of $R$ onto itself that have a (finite) derivative everywhere ([2, Corollary 2.2]). After reading these results the following question is implicit: is the same result true if $R$ is replaced by $C$, the complex numbers, and $D(R)$ is replaced by $D(C)$, the set of all entire (that is, analytic everywhere) maps from $C$ to $C$ ? It turns out that it is not true. We note that an automorphism $\psi$ of $D(C)$ is inner if there exists a bijection $h \in D(C)$, with $h^{-1} \in D(C)$, such that $\psi(f)=h \circ f \circ h^{-1}, \forall f \in D(C)$.

Before we begin we recall a result proven in [2]; that is, if 0 is a semigroup of maps from a space $X$ to itself (composition is the semigroup operation) and if $D$ contains all the constant maps from $X$ to $X$, then for every automorphism $\psi$ of $D$ there exists a unique bijection $h$ from $X$ to $X$ such that $\psi(f)=h \circ f \circ h^{-1}, \forall f \in D$.

EXAMPLE. There exists an automorphism of $D(C)$ which is not inner.

Received 11 April 1972. Communicated by S. Yamamuro. $|6|$ 
Proof. Let $h: C \rightarrow C$ be the complex conjugate function; that is if $z=x+i y$ then $h(z)=x-i y=\bar{z}$. Then it is easily checked that $\psi: D(C)+D(C)$ defined by $\psi(f)=h \circ f \circ h^{-1}, \forall f \in D(C)$, is an automorphism. Also, since $D(C)$ certainly contains all constant maps, no other $h$ represents $\psi$ in this way. But $h \neq D(C)$, so $\psi$ is not inner.

Analogous to Corollary 2.2 in [2] we have:

COROLLARY. The set of all inner cutomorphisms of $D(C)$ is isomorphic to the set of all maps in $D(C)$ of the form $f(z)=a z+b$, where $a, b \in C$ and $a \neq 0, \forall z \in C$. Call the set of all such maps $A(C)$.

Proof. First we note that the set of all entire bijections is precisely $A(C)$ (see, for example, Theorems 7.5 and 7.6 of [1], pp. 178-179). Now consider the map $\alpha$ mapping $A(C)$ onto the set of all inner automorphisms defined by, $\forall h \in A(C), \alpha(h)=\psi$, where $\psi(f)=h \circ f \circ h^{-1}, \forall f \in D(C)$. That $\alpha$ is a homomorphism onto is easily checked. That $\alpha$ is one-to-one follows from the fact that such an $h$ for a given $\psi$ is unique. So $\alpha$ is an isomorphism.

Another method of extension has been attempted; namely, to the semigroup of Frechet-differentiable maps on a real Banach space. Whether or not all automorphisms are inner or not is not yet known, but for the best result so far in this regard one should see Yamamuro [3].

\section{References}

[1] George W. Mackey, Lectures on the theory of functions of a complex variable (Van Nostrand, Frinceton, New Jersey; Toronto, Ontario; London; 1967).

[2] Kenneth D. Magill, Jr, "Automorphisms of the semigroup of all differentiable functions", Glasgow Math. J. 8 (1967), 63-66.

[3] Sadayuki Yamamuro, "On the semigroup of differentiable mappings", $J$. Austral. Math. Soc. 10 (1969), 503-510.

Department of Mathematics, University of British Columbia, Vancouver, B.C., Canada. 